\title{
A influência do protocolo de reabilitação de Cawthorne e Cooksey no equilíbrio de idosas
}

\author{
The influence of Cawthorne and Cooksey rehabilitation protocol in balance of elderly women
}

\author{
Juliana de Oliveira Souzaa, Ariela Torres Cruz ${ }^{b}$ \\ a Fisioterapeuta. Centro Universitário de Barra Mansa - UBM. Barra Mansa, RJ - Brasil. \\ b Mestre em Bioengenharia - Universidade do Vale do Paraíba - Univap. São José dos Campos, SP. Fisioterapeuta e Docente - Centro Universitário de Barra Mansa - UBM. \\ Barra Mansa, RJ - Brasil.
}

\begin{tabular}{l|l} 
RESUMO Objetivo: Este estudo teve como objetivo avaliar a influência do protocolo de reabilitação de Cawthorne e Cooksey
\end{tabular} no equilíbrio de idosas institucionalizadas.

Materiais e Métodos: Ensaio clínico controlado e randomizado. Após a verificação dos critérios de inclusão e exclusão, 10 idosas com idade entre 60 e 88 anos, foram aleatoriamente escolhidas e distribuídas em dois grupos: cinco idosas no grupo experimental (GE), submetidas ao protocolo de reabilitação de Cawthorne e Cooksey; e cinco no grupo controle (GC), que não receberam o tratamento proposto. Ambos os grupos foram avaliados antes e após três meses de tratamento através da Escala de Equilíbrio de Berg (EEB). Após a avaliação, as idosas do GE foram submetidas individualmente a 32 sessões do protocolo de reabilitação de Cawthorne e Cooksey, realizadas 2 vezes por semana, por um período de três meses, com duração de 50 minutos cada.

Resultados: Constatou-se que houve um aumento no escore da EEB do GE $(p=0,0008)$ quando comparado ao GC $(p=0,0486)$ e consequentemente diminuição da possibilidade de queda.

Conclusão: Os resultados do presente estudo mostram que o protocolo de reabilitação de Cawthorne e Cooksey foi capaz de melhorar o equilíbrio das idosas institucionalizadas participantes do estudo.

Palavras-chave: acidentes por quedas; sistema vestibular; equilíbrio postural; idoso; fisioterapia.

ABSTRACT

Objective: This study aimed to evaluate the influence of Cawthorne and Cooksey rehabilitation protocol in the balance of institutionalized elderly women.

Materials and Methods: Randomized controlled clinical trial. After checking the inclusion and exclusion criteria, 10 elderly women, aged 60 to 88 years, were randomly chosen and divided into two groups: five elderly women in the experimental group (GE), submitted the rehabilitation protocol of Cawthorne and Cooksey, and five in the control group (GC), who did not receive the proposed treatment. Both groups were evaluated before and after three months of treatment by the Berg Balance Scale (BBE). Following the evaluation, the elderly women of the GE were submitted individually to 32 sessions of rehabilitation using the protocol of Cawthorne and Cooksey, conducted 2 times per week, for a period of three months, lasting 50 minutes each.

Results: It was found that there was an increase in BBE score of the GE $(p=0.0008)$ compared to GC $(p=0.0486)$ and, consequently, a decrease in the likelihood of falls.

Conclusion: The results of this study shows that the Cawthorne and Cooksey rehabilitation protocol was able to improve the balance of institutionalized elderly women who participated in the study.

Keywords: accidental falls; vestibular system; postural balance; aged; physiotherapy. 


\section{INTRODUÇÃO}

Nos últimos anos o Brasil passou por importantes transições demográficas. A proporção de idosos que em 1999 era de $9,1 \%$ subiu para $11,3 \%$, compondo hoje um contingente acima de 22 milhões de pessoas, superando a população idosa de diversos países europeus como a França, a Inglaterra e a Itália, segundo estimativas das Nações Unidas'

A diminuição da habilidade para controlar a postura e a marcha é um dos principais problemas associados ao envelhecimento, podendo levar a ocorrência de quedas e limitações funcionais. Considerada como um importante problema de saúde pública entre os idosos, a queda pode levá-los a injúria, incapacidade e morte². Entre os idosos institucionalizados existem fatores de risco específicos que aumentam significativamente a probabilidade de ocorrência de quedas quando comparados aos idosos que vivem na comunidade, como sedentarismo, incapacidade funcional, diminuição de força muscular, flexibilidade, amplitude de movimento e função proprioceptiva das articulações em membros inferiores, instabilidade postural, vertigem, problemas visuais, demência, depressão e uso de certos medicamentos ${ }^{2,3,4}$.

As mulheres idosas apresentam maior propensão para quedas, chegando a um risco duas vezes maior do que entre os homens. Estudos sugerem que o declínio físico-funcional, o distúrbio motor e as consequências das doenças crônicas são diferentes entre homens e mulheres, contribuindo para um maior risco de quedas em mulheres do que em homens ${ }^{5}$.

O sistema vestibular, localizado na orelha interna, é responsável por promover controle postural ao corpo humano associado aos sistemas visual, somatossensorial e musculoesquelético ${ }^{6}$. Durante o processo de envelhecimento este sistema passa por processos degenerativos, que com o passar do tempo, à medida que se acentuam, podem se tornar responsáveis pela ocorrência de vertigem, tontura, zumbido e alterações de equilíbrio corporal na população idosa ${ }^{7}$.

Por estarem inseridos em um ambiente com pouca estimulação neurossensorial, os idosos institucionalizados apresentam maior prevalência de alterações vestibulares quando comparados aos idosos que vivem na comunidade ${ }^{8}$. Entre os protocolos de reabilitação vestibular encontrados na literatura, um dos mais utilizados é o de Cawthorne e Cooksey, criado na Inglaterra em 1946 e implantado no Brasil há duas décadas ${ }^{6}$. Este protocolo é constituído por exercícios que envolvem movimentos de cabeça, pescoço e olhos, exercícios de controle postural em várias posições, uso de superfície de suporte macia para diminuição do input proprioceptivo e exercícios de olhos fechados, dentre outros, com o intuito de aprimorar a interação vestibulovisual durante a movimentação cefálica e melhorar a estabilidade postural estática e dinâmica9 ${ }^{9}$.

Cawthorne e Cooksey iniciaram suas pesquisas atendendo pacientes portadores de alteração do equilíbrio pós-traumatismo cranioencefálico e, atualmente, de acordo com a literatura, a aplicação do protocolo de reabilitação proposto pelos autores vem apresentando bons resultados em diversos pacientes com alterações do equilíbrio decorrentes de diferentes causas ${ }^{6,9}$.

Assim, considerando que a queda é um evento que modifica substancialmente a qualidade de vida do idoso e que a população idosa vem aumentando a cada ano, este estudo teve como objetivo avaliar a influência do protocolo de reabilitação de Cawthorne e Cooksey no equilíbrio de idosas institucionalizadas.

\section{MATERIAIS E MÉTODOS}

Este ensaio clínico controlado e randomizado foi desenvolvido em uma Instituição de Longa Permanência (ILP) no estado do Rio de Janeiro após aprovação do Comitê de Ética em Pesquisa (CEP) do Centro Universitário de Barra Mansa (UBM), sob protocolo no 009/2014, respeitando todos os princípios éticos que norteiam a pesquisa, bem como a privacidade de seus conteúdos, como preconizam os documentos internacionais e a Resolução 466/12 do Conselho Nacional de Saúde do Ministério da Saúde.

Os critérios de inclusão foram: mulheres com idade igual ou superior a 60 anos, registradas como moradoras da ILP, com uma adequada compreensão e competências de comunicação, com escore abaixo de 56 pontos na Escala de Equilíbrio de Berg (EEB) e que não estivessem sob outro tratamento para melhora do equilíbrio. O sexo feminino foi escolhido porque estudos mostram que há uma proporção maior de mulheres institucionalizadas, do que homens, sendo que o risco de queda também é maior entre elas ${ }^{10,11}$.

Os critérios de exclusão foram: idosas que apresentassem doenças que interferissem no equilíbrio (labirintite, neuropatias, cerebelopatias, vestibulopatias, doença de Parkinson, acidente vascular encefálico (AVE), esclerose múltipla, dentre outras.), que dependessem de dispositivos auxiliares para a locomoção (bengala, muleta ou andador) ou cadeirantes, as que estivessem restritas ao leito, que apresentassem distúrbios visuais e/ou auditivos não corrigidos e alterações musculoesqueléticas e cognitivas que impedissem a realização adequada da avaliação e do protocolo de reabilitação de Cawthorne e Cooksey.

Após sorteio aleatório realizado pelas próprias pesquisadoras, 10 idosas foram distribuídas em dois grupos: 
cinco idosas no grupo experimental (GE), submetidas ao protocolo de reabilitação de Cawthorne e Cooksey; e cinco no grupo controle (GC), que não receberam o tratamento proposto.

Ambos os grupos foram submetidos a duas avaliações por meio da EEB antes do início do tratamento e três meses após a primeira avaliação, utilizando os seguintes equipamentos: duas cadeiras (uma com braços e outra sem braços), um cronômetro, uma fita métrica e um banquinho. Para cada tarefa, foram dadas instruções por meio de comando verbal e se necessário, a atividade era demonstrada para as idosas.

A EEB foi criada em 1992 por Berg ${ }^{12}$ e adaptada para a língua portuguesa em $2004^{13}$. A escala, que pode ser facilmente administrada, avalia o equilíbrio estático e dinâmico baseada em 14 itens e com pontuação máxima de 56. Cada item possui uma escala ordinal de cinco alternativas que variam de 0 a 4 pontos. A capacidade em realizar a atividade de modo independente é expressa na escala através da pontuação 4; quando há necessidade de supervisão por parte do avaliador a pontuação é 3; quando o indivíduo necessita de segurança e auxílio moderado a pontuação é 2; quando há necessidade de auxílio e o tempo de permanência na postura de equilíbrio é mínima a pontuação é 1 e se o indivíduo for incapaz de realizar a tarefa de maneira independente a pontuação é 0 . Assim, quanto menor for à pontuação, maior é o risco de quedas ${ }^{12}$.

De acordo com Chiu et al. ${ }^{14}$ entre os escores 56 e 54, cada ponto a menos na EEB está associado a um aumento de 3 a $4 \%$ no risco de quedas e entre 54 e 46 , há um aumento de 6 a $8 \%$ de chances. O escore de 45 pontos permite separar indivíduos com risco baixo e moderado de quedas e escore igual ou menor que 36 está associado a 100\% de risco de quedas.

Os elementos que compõem a escala são representativos de atividades da vida diária: sentado para em pé; em pé sem apoio; sentado sem apoio com os pés no chão; em pé para sentado; realizando transferências, em pé sem apoio com os olhos fechados; em pé sem ajuda com os pés juntos; inclinado para frente com um membro superior estendido; pegando objetos do chão; olhando para trás sobre os ombros direito e esquerdo; girando $360^{\circ}$; pisando em um banquinho alternando os pés; em pé sem apoio com um dos pés à frente e em pé sobre apenas um dos membros $^{13}$.

Após a avaliação inicial, as idosas do GE foram submetidas individualmente a 32 sessões do protocolo de reabilitação de Cawthorne e Cooksey, realizadas 2 vezes por semana, por um período de três meses, com duração de 50 minutos cada. Trata-se basicamente de exercícios de movimentação de cabeça, olhos, tronco, marcha, associados ou não, com ou sem apoio visual, proprioceptivo, tátil, entre outros. Este protocolo foi escolhido pelas pesquisadoras devido à simplicidade de aplicação dos exercícios e seu baixo custo. Cabe ressaltar que, a aplicação da EEB e do protocolo de reabilitação foi realizada pela própria pesquisadora previamente treinada.

Os dados coletados foram exportados para um sistema de banco de dados e analisados com auxílio do programa BioEstat versão 5.0. Para verificar se eles seguiam uma distribuição normal, foi realizado o teste de normalidade de Shapiro-Wilk, no qual os valores apresentados foram considerados normais. Nesse contexto, foi aplicado o teste paramétrico " $\mathrm{t}$ " de Student para observações pareadas (dependentes) e não pareadas (independentes), com nível de significância de $p \leq 0,05$.

\section{RESULTADOS}

As 23 idosas da ILP aceitaram participar desta pesquisa. Dentre elas, 56\% (13 idosas) não participaram, pois se enquadraram nos critérios de exclusão. As porcentagens foram as seguintes: $9 \%$ (2 idosas) eram cadeirantes, 13\% (3 idosas) dependiam de dispositivos auxiliares para a locomoção, 13\% (3 idosas) apresentavam déficits cognitivos, 4\% (1 idosa) apresentava úlcera varicosa em membro inferior esquerdo, 9\% (2 idosas) apresentavam déficit visual não corrigido, 4\% (1 idosa) estava acamada por fratura de fêmur e 4\% (1 idosa) acamada por sequela de AVE. Assim, foram selecionadas 10 idosas aptas a participarem da pesquisa, com idade entre 60 e 88 anos (Figura 1). Quanto à faixa etária da amostra total, verificou-se que $50 \%$ das idosas tinham de 60 a 69 anos, $20 \%$ de 70 a 79 anos e $30 \%$ de 80 a 88 anos. A idade média para o GE foi de 74,80 anos $( \pm 9,03)$ e para o GC foi de 69,20 anos $( \pm 11,12)$.

Os resultados obtidos mostram que o protocolo de reabilitação de Cawthorne e Cooksey promoveu melhora do equilíbrio das participantes do estudo, pois verificou-se um valor de $p=0,0008$ para o GE e $p=0,0486$ para o GC. Ao compararmos os grupos, observa-se que o GE teve resultados significativos quando comparado ao GC $(p=0,0033)$ (Figura 2).

De acordo com a interpretação dos escores da EEB das participantes do estudo, durante a 1a avaliação, 100\% $(n=5$ idosas) do GE apresentou um alto risco para a ocorrência de quedas, e após 3 meses 100\% ( $n=5$ idosas) apresentou risco moderado. Na 1a avaliação do GC $40 \%$ ( $n=2$ idosas) apresentou um alto risco para a ocorrência de queda e $60 \%$ ( $n=3$ idosas) um risco moderado, e após 3 meses, 80\% ( $n=4$ idosas) apresentou alto risco e apenas $20 \%$ ( $n=1$ idosa) um risco moderado (Tabela 1). 

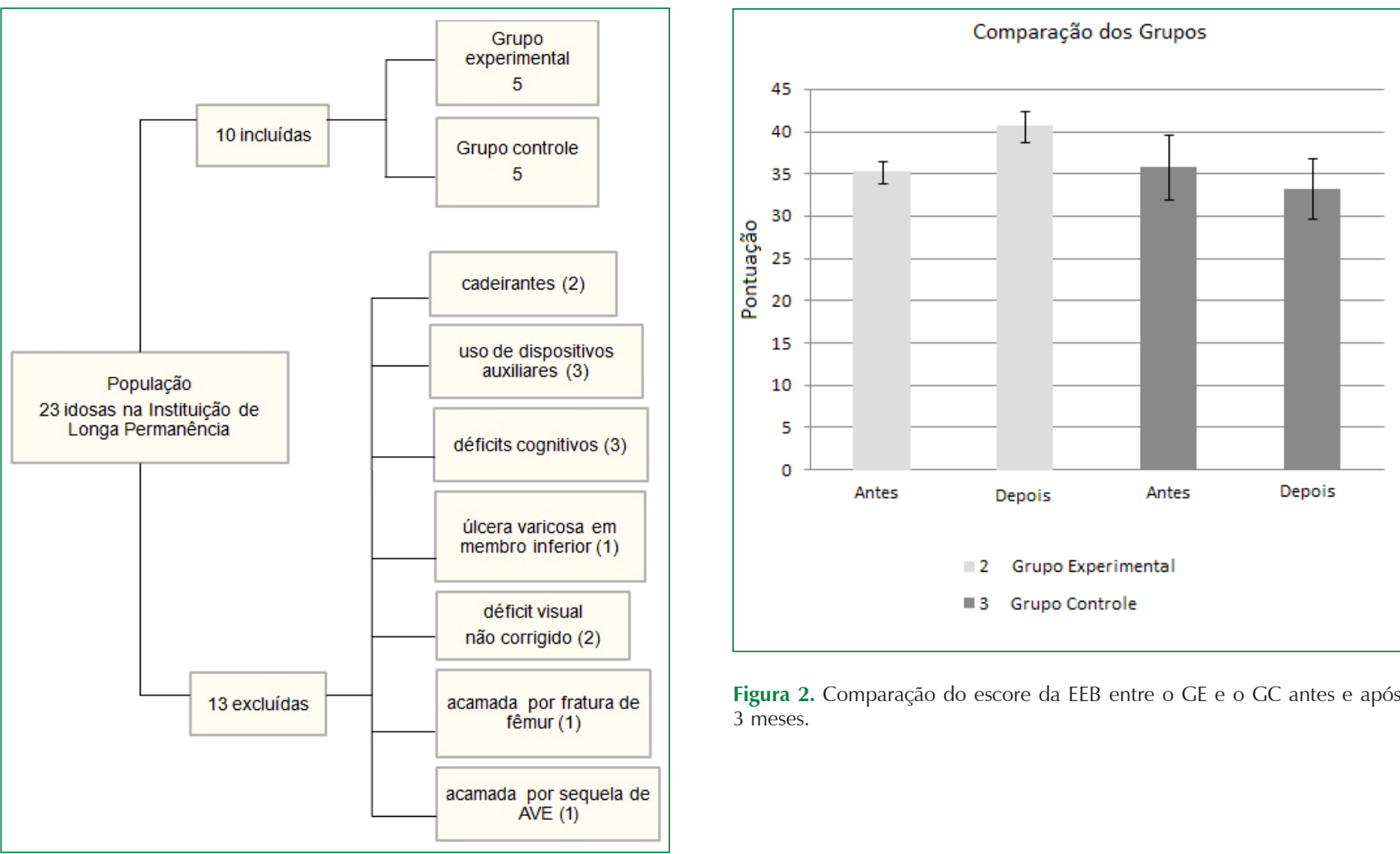

Figura 2. Comparação do escore da EEB entre o GE e o GC antes e após 3 meses.

Figura 1. Fluxograma do processo de seleção para participação no estudo.

Tabela 1. Comparação do risco de ocorrência de queda entre o GE e o GC antes e após 3 meses, de acordo com o escore da EEB.

\begin{tabular}{lcccc}
\hline & \multicolumn{2}{c}{ Grupo Experimental } & \multicolumn{2}{c}{ Grupo Controle } \\
\cline { 2 - 5 } Risco para ocorrência de queda & Antes & Após 3 meses & Antes & Após 3 meses \\
\cline { 2 - 5 } & $\mathbf{N}(\%)$ & $\mathbf{N}(\%)$ & $\mathbf{N}(\%)$ & $\mathbf{N}(\%)$ \\
Alto Risco & $5(100)$ & $0(0)$ & $2(40)$ & $4(80)$ \\
Risco Moderado & $0(0)$ & $5(100)$ & $3(60)$ & $1(20)$ \\
Baixo Risco & $0(0)$ & $0(0)$ & $0(0)$ & $0(0)$ \\
\hline
\end{tabular}

\section{DISCUSSÃO}

Lopes et al. ${ }^{3}$ apontam em seu estudo que quanto maior a idade média dos participantes, maior é o risco de quedas. De acordo com Peixoto et al. ${ }^{15}$ a prevalência de quedas aumenta em idosos acima do 80 anos, sendo que em idosos institucionalizados esta prevalência é consideravelmente maior. Pesquisadores apontam que independente da idade a institucionalização por si só, gera incapacidades, tornandose um fator de risco para quedas, sendo necessários atenção e cuidados especiais ${ }^{16}$.

Verificou-se que as idosas com 60 anos, participantes do estudo, já apresentavam baixos escores na EEB e que não havia idosas com baixo risco para ocorrência de quedas.
Entretanto, não há um consenso na literatura sobre a faixa etária na qual os idosos começam a apresentar déficits no controle do equilíbrio corporal.

A literatura aponta uma variedade de testes com o objetivo de avaliar o controle do equilíbrio corporal e estabelecer parâmetros para a identificação de idosos com maior risco de sofrerem quedas ${ }^{17}$. Porém, autores apontam que EEB apresenta maior confiabilidade e validade quando comparada a outros instrumentos de avaliação. Ressaltase ainda que a EEB avalia muitos aspectos diferentes do equilíbrio e necessita de poucos equipamentos para ser administrada, em relação às demais ${ }^{18}$.

Os resultados obtidos e publicados pela literatura mundial vêm incentivando os pesquisadores a utilizarem o método 
de reabilitação vestibular, considerada uma excelente opção terapêutica, pois, além de melhorar o equilíbrio do indivíduo, tem função profilática, ajudando-o a restabelecer a confiança em si mesmo, reduzindo a ansiedade e melhorando o convívio social ${ }^{18}$. Ainda que os idosos não possuam diagnóstico clínico de disfunção vestibular, eles apresentam alterações no equilíbrio e têm o risco de sofrerem quedas ${ }^{19}$. No estudo de Santos et al. ${ }^{16}$ verificou-se que $100 \%$ dos idosos institucionalizados, participantes do estudo, apresentaram risco de quedas, concordando com os achados do presente estudo.

Autores sugerem que, embora não seja esperada uma completa recuperação funcional do equilíbrio, a utilização da reabilitação vestibular é conduta adequada, e seus resultados devem ser interpretados como positivos em função das expectativas do terapeuta e do paciente ${ }^{18}$. Os resultados de um estudo com 21 idosos mostraram que após a reabilitação vestibular houve uma evolução nas habilidades para execução dos testes relativos ao equilíbrio estático e dinâmico, utilizando a $\mathrm{EEB}^{20}$.

Autores afirmam que indivíduos idosos que apresentam instabilidade postural, devem ser submetidos a protocolos de reabilitação vestibular, como os de Cawthorne e Cooksey ${ }^{19}$. Sugere-se que a plasticidade neuronal é estimulada com a aplicação desse protocolo e isto possibilita a recuperação de funções neurológicas centrais relacionadas ao controle do equilíbrio corporal. Tais mecanismos relacionados à plasticidade neuronal do sistema nervoso central promovem a estabilização visual durante os movimentos da cabeça, melhorando a interação vestíbulo-visual durante a movimentação cefálica, ampliando a estabilidade postural estática e dinâmica, em condições que produzem informações sensoriais conflitantes ${ }^{21}$.

O período de aplicação dos exercícios e das sessões varia amplamente entre os estudos, tornando-se difícil estabelecer o melhor protocolo, tempo de intervenção, entre outras variáveis. No atual estudo os exercícios de Cawthorne e Cooksey foram aplicados duas vezes por semana, com duração de 50 minutos, durante três meses. De acordo com a literatura a aplicação dos exercícios tem sido feita entre duas a três vezes na semana, com duração de 45 a 60 minutos, durante dois a três meses, sendo capazes de promover bons resultados no que diz respeito à melhora do equilíbrio e a diminuição da possibilidade de quedas em idosos ${ }^{20,21}$.

No atual estudo constatou-se que mesmo após serem submetidas aos exercícios, as idosas do GE tiveram dificuldades para realizar algumas tarefas da EEB como: inclinar-se para frente com um membro superior estendido, pisar alternadamente em um banquinho, ficar em pé sem apoio com um dos pés à frente e ficar em pé sobre apenas um dos membros. Isso pode ser explicado, pois durante o processo de envelhecimento ocorrem diversas alterações na estrutura corporal, como a perda da flexibilidade e da força muscular em membros inferiores, superiores e tronco, dificultando a execução de tais tarefas ${ }^{22}$. Além disso, exercícios para o aumento da flexibilidade e força muscular não foram realizados neste estudo.

De acordo com Santana et al. ${ }^{23}$, para manter o equilíbrio corporal, torna-se necessário apresentar, além de integridade do sistema sensorial e nervoso, preservação das condições normais do sistema musculoesquelético. Dentre os sistemas que controlam o equilíbrio corporal, o sistema musculoesquelético tem forte contribuição para a manutenção desta função.

De acordo com Jonsson et al..$^{24}$ a tarefa de ficar em pé sobre apenas um dos membros, por exemplo, requer uma ação voluntária inicial de mover o centro de gravidade sobre o membro apoiado (fase dinâmica), seguida da tarefa de manter a orientação postural no espaço (fase estática). Autores afirmam que os idosos apresentam dificuldades para ajustar a posição unipodal na fase dinâmica e consequentemente dificuldades em manter o apoio unipodal na fase estática. Eles ainda sugerem que o tempo de permanência em apoio unipodal está relacionado à diminuição da força muscular que ocorre com o envelhecimento ${ }^{25}$.

Assim, autores afirmam que o programa de reabilitação para melhora do equilíbrio de idosos deve incluir além dos exercícios vestibulares, treinos específicos de equilíbrio, fortalecimento muscular com ênfase em tronco e membros inferiores e estimulação proprioceptiva, optando por uma escolha individual de reabilitação de acordo com as necessidades de cada indivíduo ${ }^{26,27}$.

Como limitação do estudo destaca-se o reduzido tamanho amostral. Miot ${ }^{28}$ afirma que se o tamanho da amostra do estudo for muito restrito $(<30)$, é dificultada a análise de subgrupos e comprometido o desempenho dos testes estatísticos. Considera-se também como fator limitante a falta de outros recursos terapêuticos na intervenção do estudo. Diante dessas limitações, sugere-se que sejam realizados novos estudos que abordem a mesma temática, com um número maior de participantes e com um terceiro grupo que seja submetido a outros recursos e técnicas que também visem à melhora do equilíbrio, como por exemplo, alongamentos, fortalecimento muscular e estimulação proprioceptiva.

Os resultados deste estudo demonstraram que as idosas apresentavam risco para a ocorrência de quedas. Após três meses de intervenção as idosas do grupo experimental obtiveram melhora no índice da EEB quando comparadas ao grupo controle, sendo que o fator idade não foi considerado limitante sobre a resposta final do tratamento. Portanto, 
conclui-se que o protocolo de reabilitação de Cawthorne e Cooksey foi capaz de melhorar o equilíbrio das idosas institucionalizadas participantes do estudo e, podendo assim, diminuir a possibilidade de quedas.

\section{REFERÊNCIAS}

1. Minayo MCS. O envelhecimento da população brasileira e os desafios para o setor da saúde. Cad Saúde Pública 2012;28(2): 208-9. http://dx.doi.org/10.1590/S0102-311X2012000200001

2. Alves NB, Scheicher ME. Equilíbrio postural e risco para queda em idosos da cidade de Garça, SP. Rev Bras Geriatr Gerontol. 2011;14 (4):763-8. http://dx.doi.org/10.1590/S1809-98232011000400015

3. Lopes MNSS, Passerini CG, Travensolo CF. Eficácia de um protocolo fisioterapêutico para equilíbrio em idosos institucionalizados. Semina Cienc Biol Saude. 2010;31(2):143-52. http://dx.doi. org/10.5433/1679-0359.2011v32n2p143

4. Duca GFD, Antes DL, Hallal PC. Quedas e fraturas entre residentes de instituições de longa permanência para idosos. Rev Bras Epidemiol. 2013;16(1):68-76. http://dx.doi.org/10.1590/S1415790X2013000100007

5. Soares WJS, De Moraes AS, Ferriolli E, Perracini MR. Fatores associados a quedas e quedas recorrentes em estudo: estudo de base populacional. Rev Bras Geriatr Gerontol. 2014;17(1):49-60. http://dx.doi.org/10.1590/S1809-98232014000100006

6. Onishi ET, Kasse CA, Barreiro FCAB, Doná F. Avaliação e reabilitação do equilíbrio corporal: abordagem interdisciplinar. São Paulo: Copyrigh; 2013.

7. Scherer S, Lisboa HrK, Pasqualotti A. Tontura em idosos: diagnóstico otoneurológico e interferência na qualidade de vida. Rev Soc Bras Fonoaudiol. 2012;17(2):142-50. http://dx.doi.org/10.1590/S151680342012000200007

8. Shimizu WAL, Uematsu ESC, Peterlin CB, Brito RMS. Prevalência de sinais e sintomas de disfunção vestibular em idosos institucionalizados e não institucionalizados. Med Reabil. 2010;29(2):52-6.

9. Ricci NA, Aratani MC, Doná F, Macedo C, Caovilla HH, Ganança FF. Revisão sistemática sobre os efeitos da reabilitação vestibular em adultos de meia idade e idosos. Rev Bras Fisioter. 2010;14(5): 361-71. http://dx.doi.org/10.1590/S1413-35552010000500003

10. Ferreira DCO, Yoshitome AY. Prevalência e características das quedas de idosos institucionalizados. Rev Bras Enferm. 2010;63(6):991-7. http://dx.doi.org/10.1590/S0034-71672010000600019

11. Cunha AA, Lourenço RA. Quedas em idosos: prevalência e fatores associados. Revista HUPE. 2014;13(2):21-9. http://dx.doi. org/10.12957/rhupe.2014.10128

12. Berg KO. Clinical and laboratory measures of postural balance in an elderly population. Arch Phys Med Rehabil. 1992;73(11):1073-80.

13. Miyamoto ST, Lombardi Junior I, Berg KO, Ramos LR, Natour J. Brazilian version of the Berg balance scale. Braz J Med Biol Res. 2004;37(9):1411-21. http://dx.doi.org/10.1590/S0100879X2004000900017
14. Chiu AYY, Au-Yeung SSY, Lo SK. A comparison of four functional tests in discriminating fallers from non-fallers in older people. Disabil Rehabil. 2003;25(1):45-50. http://dx.doi.org/10.1080/ dre.25.1.45.50

15. Peixoto TM, Artelosa RCC, Silva LAT, Santos TSM. Causas e conseqüências de quedas em idosos atendidos no hospital de Santo Estevão, BA. Biociências. 2015;21(2):93-100.

16. Santos MV, Weizenmann SE, Koetz LCE. Avaliação dos idosos e a percepção dos profissionais quanto aos riscos de quedas em uma instituição de longa permanência. Cinergis. 2015;16(1):2-6.

17. Karuka AH, Silva JAMG, Navega MT. Análise da concordância entre instrumentos de avaliação do equilíbrio corporal em idosos. Rev Bras Fisioter. 2011;15(6):460-6. http://dx.doi.org/10.1590/S141335552011000600006

18. Neto JP, Raso W, Brito CAF. Mobilidade funcional em função da força muscular em mulheres idosas fisicamente ativas. Rev Bras Med Esporte. 2015;21(5):369-71. http://dx.doi.org/10.1590/1517869220152105112756

19. Batista JS, Pasqualotti A, Marchi ACB, Wibelinger LM. A reabilitação vestibular e o envelhecimento humano. Rev Bras Ciênc Saúde. 2011;9(27):57-63.

20. Peres $M$, Silveira E. Efeito da reabilitação vestibular em idosos: quanto ao equilíbrio, qualidade de vida e percepção. Ciênc Saúde Coletiva. 2010;15(6):2805-14. http://dx.doi.org/10.1590/S141381232010000600018

21. Castro DLS, Pontes NG. Proposta de tratamento fisioterapêutico na reabilitação vestibular para pacientes com vertigem posicional paroxística benigna [monografia]. Belém: Universidade da Amazônia; 2008.

22. Oliveira ESAO, Costa JCB, Raphael SB, Guabiroba JS, Silva IP. Idosos e exercício físico: a aderência da prática como fator predominante para wellness. Rev Int Cienc Deporte. 2015;11(2):117-8.

23. Santana FJ, Lima MCAML. Prevalência de quedas e dor musculoesquelética em idosos. Saúde \& Amb Rev. 2014;3(2): 80-9.

24. Jonsson E, Seiger A, Hirschfeld H. One-leg stance in healthy young and elderly adults: a measure of postural steadiness. Clin Biomech (Bristol, Avon). 2004;19(7):688-94. http://dx.doi.org/10.1016/j. clinbiomech.2004.04.002

25. Dias BB, Mota RS, Gênova TC, Tamborelli V, Pereira VV, Puccini PT. Aplicação da Escala de Equilíbrio de Berg para verificação do equilíbrio de idosos em diferentes fases do envelhecimento. Rev Bras de Ciên do Envelh Hum. 2009;6(2):213-24. http://dx.doi. org/10.5335/rbceh.2009.020

26. Delissa JA. Tratado de Medicina da Reabilitação. $2^{\underline{a}}$ ed. São Paulo: Editora Manole; 2002.

27. Alfieri FM, Teodori RM, Guirro RRJ. Estudo baropodométrico em idosos submetidos à intervenção fisioterapêutica. Fisioter Mov. 2006;19(2):67-74.

28. Miot HÁ. Tamanho da amostra em estudos clínicos e experimentais. J Vasc Bras. 2011;10(4):p. 275-8. 